\title{
Academias ao ar livre: expectativa ou realidade na promoção de saúde?
}

\author{
Outdoor gyms: expectation or reality in health promotion?
}

\author{
Pollyanna Natalia Micali ${ }^{1}$ \\ Raiana Lídice Mór Fukushima² \\ Elisangela Gisele do Carmo ${ }^{3}$ \\ José Luiz Riani Costa ${ }^{4}$ \\ Jamile Sanches Codogno
}

\section{Endereço para Correspondência:}

Pollyanna Natalia Micali

Avenida 44-A, 995, apto-1, Vila Nova

13.506-610 - Rio Claro, SP [Brasil]

pollymicali@gmail.com

\begin{abstract}
Resumo
Introdução: o Programa Academia da Saúde, objetiva promover qualidade de vida e reduzir a vulnerabilidade e riscos à saúde.

Objetivo: o presente estudo buscou identificar os fatores associados ao uso das academias ao ar livre (AAL), estratificados por sexo e idade, bem como, traçar o perfil desses usuários em relação aos fatores sociodemográficos e as condições de saúde. Métodos: trata-se de um estudo transversal, realizado em 2015 com o seguinte procedimento de amostragem: seleção de setores censitários; seleção de domicílios; seleção de residentes.

Resultados: a amostra foi composta por 671 participantes, acima de 26 anos, apontando que $67,99 \%$ das mulheres e $60,99 \%$ dos homens conhecem as AAL. Sobre fazer ou já ter feito uso desse espaço, 16,39\% da amostra total relataram fazer ou já ter feito uso. Entre as mulheres, $15,18 \%$ fazem ou já fizeram uso desse espaço, comparadas a $18,51 \%$ dos homens.

Conclusão: dessa forma, foram discutidas alternativas que viabilizem maior aderência e utilização desses espaços públicos.
\end{abstract}

Descritores: Academia ao ar livre. Saúde pública. Promoção da saúde.

\begin{abstract}
Introduction: The Health Academy Program aims to promote quality of life and reduce vulnerability and health risks.

Objective: This study aimed to identify the factors associated with the use of open air academies (AAL), stratified by sex and age, as well as to outline the profile of these users in relation to sociodemographic factors and health conditions.

Methods: This is a cross-sectional study carried out in 2015 with the following sampling procedure: selection of census tracts; household selection; selection of residents.

Results: The sample consisted of 671 participants, over 26 years old, indicating that $67.99 \%$ of the women and $60.99 \%$ of the men know about AAL. On making or having already made use of this space, $16.39 \%$ of the total sample reported to do or have already made use. Among women, $15.18 \%$ make or have used this space, compared to $18.51 \%$ of men.

Conclusion: In this way, alternatives were discussed that allow greater adhesion and use of these public spaces.
\end{abstract}

Keywords: Outdoor gym. Public health. Health promotion.

\section{Cite como}

Micali PN, Fukushima RLM, Carmo EGdo, Costa JLR, Codogno JS.

Academias ao ar livre: expectativa ou realidade na promoção de saúde? Conscientiae Saúde 2019 jul./set.; 18(3):376-388 https://doi.org/10.5585/ConsSaude.v18n3 13380. 


\section{Introdução}

Em Ottawa (1986), ocorreu a I Conferência Internacional sobre Promoção da Saúde, a fim de contribuir para o "Saúde para Todos", o qual teve início em 2000. Nesta ocasião, foi apresentada a "Carta de Intenções" tendo como caráter formular respostas às crescentes expectativas por uma nova saúde pública, movimento que aconteceu em todo o mundo ${ }^{1}$ Tal documento, ainda afirmou que os pré-requisitos e perspectivas para a saúde, não são assegurados somente pelo setor da saúde, apontando que a promoção da saúde demanda uma ação coordenada intersetorial entre o governo, o setor da saúde e outros setores sociais e econômicos, assim como, organizações voluntárias e não-governamentais, autoridades locais, indústrias e mídia. As estratégias e programas na área da promoção da saúde devem se adaptar às necessidades locais e às possibilidades de cada país e região, bem como levar em conta as diferenças em seus sistemas sociais, culturais e econômicos ${ }^{1}$.

Diante do exposto, a Política Nacional de Promoção da Saúde e o Plano de Enfrentamento das Doenças e Agravos Não-Transmissíveis, desenvolveram ações que embasaram a criação do Programa Academia da Saúde, objetivando promover qualidade de vida e reduzir a vulnerabilidade e riscos à saúde. Dessa forma, o Ministério da Saúde instituiu o Programa Academia da Saúde no âmbito do Sistema Único de Saúde (2011), para ser implantado pelas Secretarias de Saúde dos Municípios, tendo a promoção da atividade física (AF) como eixo central ${ }^{2}$. Alguns estudos têm demonstrado associações positivas entre a disponibilidade de equipamentos dentro dos espaços públicos e níveis mais elevados de AF. Esta evidência tem demonstrado a importância dos espaços públicos abertos para aumentar os níveis de $\mathrm{AF}$ na comunidade ${ }^{3}$. O ambiente pode proporcionar um cenário mais favorável para o aumento da $\mathrm{AF}$, propiciando os benefícios da mesma para a saúde ${ }^{4}$. Dessa forma, as academias ao ar livre (AAL) vem sendo considerada uma alternativa plausível para ser implementada nesses espaços (parques e praças). Elas são equipadas com equipamentos que se utilizam do peso corporal como carga de exercício, na intenção de melhorar os componentes da capacidade física dos usuários ${ }^{5}$.

Atualmente, o município de Rio Claro-SP conta com nove AAL, devidamente instaladas em áreas abertas para o uso da população a qualquer hora do dia ${ }^{6}$. Porém, ainda é desconhecido o perfil dos usuários que utilizam esses espaços e as possíveis barreiras que impedem ou dificultam a utilização dos não usuários. Portanto, o presente estudo buscou identificar os 
fatores associados ao uso das academias ao ar livre, estratificados por sexo e idade, bem como, traçar o perfil desses usuários em relação aos fatores sociodemográficos e as condições de saúde.

\section{Métodos}

Inicialmente, todos os participantes assinaram um termo de consentimento prévio à participação, aprovado pelo Comitê de Ética e Pesquisa da Universidade Estadual Paulista (Unesp), sob o número do Protocolo 24164813.4.0000.5465 com o parecer 626.162. Os princípios éticos também foram assegurados aos entrevistados, de acordo com a Resolução 466/2012 do Conselho Nacional de Saúde.

Este é um estudo transversal, de natureza quantitativa realizado no município de Rio Claro-SP, localizado no sudeste do Estado de São Paulo, tendo a população estimada em 198.413 habitantes ${ }^{7}$.

O procedimento de amostragem foi realizado da seguinte forma: a) seleção de setores censitários (total de 200), sendo selecionados apenas os setores ímpares (total de 99 setores) de acordo com o censo (2010); b) seleção de domicílios; c) seleção de residentes.

O presente estudo realizado em 2015 incluiu na amostra adultos com 26 anos ou mais de ambos os sexos, não institucionalizados (hospitais, instituições de longa permanência - asilos e prisões) e não apresentando doenças ou problemas de saúde que não permitiria realizar AF na semana anterior à entrevista. Dessa forma, 671 participantes foram contatados e entrevistados com êxito.

\section{Instrumentos de coleta}

Todas as entrevistas foram realizadas nas residências dos participantes por meio de um questionário eletrônico (tablets) que executa o aplicativo Open Data Kit (ODK) buscando as seguintes informações:

1) Para a caracterização da amostra, foi utilizado o questionário estruturado composto por dados sociodemográficos: ano de nascimento/idade; sexo; escolaridade e estado civil. 
2) Para acessar a classificação social foi utilizado o questionário proposto pela Associação Brasileira de Empresas de Pesquisa (ABEP), reunindo pessoas nas classes sociais A (alta), B e C (média), D (baixa).

3) Para acessar informações sobre as AAL, foi aplicado o System for Observing Play and Recreation in Communities (SOPARC), instrumento utilizado para avaliar parques e espaços públicos abertos ${ }^{8}$. O SOPARC foi aprimorado a partir de um outro instrumento disponibilizado para jovens denominado SOPLAY, tendo como diferencial classificar a área dos participantes segundo o critério de atividade física da faixa etária e etnia, dentro de um contexto comunitário onde são observados as atividades dentro dos esportes, fitness, jogos esportivos? e hábitos sedentários ${ }^{8}$. O outro instrumento utilizado na coleta foi o Questionário Internacional de Atividade física (IPAQ), versão longa, afim de investigar o nível de AF no lazer ${ }^{9}$. O IPAQ é um instrumento bem conceituado e validado em 12 países e outros 14 instituições acadêmicas, permitindo estimar o tempo gasto semanalmente pelo indivíduo em sua atividade física (moderada e vigorosa) em diversos âmbitos, seja no trabalho, no transporte, nas atividades rotineiras domésticas, atividades do contexto do lazer, assim como aquelas classificadas como passivas, como sentar-se. O instrumento possui duas versões, sendo a curta (7 questões) e a longa (27 questões).

\section{Análises Estatísticas}

Foi realizada análise descritiva das variáveis categóricas e seus resultados foram apresentados em números absolutos e percentuais. Na sequência foram adotados os testes de qui-quadrado e exato de Fisher para a comparação das variáveis categóricas entre os que usam e não usam as AAL. E para analisar os fatores associados com o uso das AAL foi utilizada a análise de regressão logística univariada e multivariada, com critério Stepwise de seleção de variáveis.

As análises estatísticas foram realizadas utilizando o Statistical Analysis System (SAS), versão $9.2^{10}$, sendo considerando $\mathrm{p}<0,05$ como nível de significância para todos os testes. 


\section{Resultados}

De acordo com a análise descritiva do presente estudo, a maior parte da amostra, composta por 671 participantes, teve maior frequência entre: mulheres $(63,79 \%)$, casados $(60,36 \%)$, escolaridade de $2^{\circ}$ grau completo $(46,5 \%)$, classe econômica C (mediana) $(68,61 \%)$ e nível de AF insuficiente $(72,43 \%)$. Da amostra total, 85,09\% dos participantes relataram ter algum tipo de DCNT (hipertensão, diabetes, depressão, asma, doença pulmonar obstrutiva, problemas cardíacos e osteomusculares), sendo que $87,14 \%$ das participantes mulheres relataram ter uma ou mais DCNT comparadas a $81,48 \%$ dos homens.

Em relação as variáveis sobre as AAL, 67,99\% das mulheres disseram conhecer este espaço, comparadas a 60,99\% dos homens. Sobre fazer ou já ter feito uso desse espaço, apenas $16,39 \%$ da amostra total relataram fazer ou já ter feito uso. Entre as mulheres, 15,18\% fazem ou já fizeram uso desse espaço, comparadas a 18,51\% dos homens.

A tabela 1, apontou a diferença entre as usuárias e não usuárias das AAL, sendo que a maior frequência de mulheres ativas está entre as não usuárias $(74,10 \%)$ e de inativas entre usuárias (86,15\%), de acordo com o nível de AF no lazer. Sobre conhecer as AAL, 62,26\% das não usuárias, não conhecem as AAL.

Tabela 1 - Associação entre o uso das AAL e os fatores sociodemográficos e as condições de saúde das mulheres ( $\mathrm{n}=428$, Rio Claro-SP, Brasil)

\begin{tabular}{|c|c|c|c|c|}
\hline Variáveis & $\begin{array}{l}\text { Não } \\
\text { N (\%) }\end{array}$ & $\begin{array}{l}\text { Sim } \\
\mathrm{N}(\%)\end{array}$ & $\mathrm{x}^{2}$ & p-valor \\
\hline Faixa Etária & & & 2,58 & 0,27 \\
\hline 25-39 anos & $49(13,50)$ & $5(7,69)$ & & \\
\hline $40-59$ anos & $146(40,22)$ & $24(36,92)$ & & \\
\hline$\geq 60$ anos & $168(46,28)$ & $36(55,38)$ & & \\
\hline Escolaridade & & & 8,72 & 0,06 \\
\hline Até a $4^{\circ}$ série & $148(40,77)$ & $30(46,15)$ & & \\
\hline $2^{\circ}$ grau incompleto & $54(14,88)$ & $7(10,77)$ & & \\
\hline $2^{\circ}$ grau completo/superior & $161(44,36)$ & $28(43,07)$ & & \\
\hline Classe Econômica (ABEP) & & & - & 0,69 \\
\hline $\mathrm{A}$ e B & $40(16,62)$ & $7(14)$ & & \\
\hline $\mathrm{C}$ & $172(69,92)$ & $34(68)$ & & \\
\hline $\mathrm{D}$ & $34(13,62)$ & $9(18)$ & & \\
\hline AF no lazer (IPAQ) & & & 4,38 & 0,03 \\
\hline Ativo & $94(25,90)$ & $9(13,85)$ & & \\
\hline Inativo & $269(74,10)$ & $56(86,15)$ & & \\
\hline
\end{tabular}




\begin{tabular}{|c|c|c|c|c|}
\hline Possui DCNT & & & 0,001 & 1,00 \\
\hline Não & $20(30,20)$ & $45(69,20)$ & & \\
\hline Sim & $262(69,80)$ & $111(30,60)$ & & \\
\hline Conhece as AAL & & & 84,57 & 0,001 \\
\hline Não & $137(37,74)$ & $0(0,00)$ & & \\
\hline Sim & $226(62,26)$ & $65(100)$ & & \\
\hline Necessário para o uso das AAL & & & 5,20 & 0,39 \\
\hline Companhia & $9(2,4)$ & $3(4,62)$ & & \\
\hline Tempo & $39(10,74)$ & $12(18,46)$ & & \\
\hline Não tem interesse & $90(24,79)$ & $15(23,08)$ & & \\
\hline Orientação profissional & $76(20,94)$ & $13(20)$ & & \\
\hline Outros Fatores & $149(41,04)$ & $22(33.85)$ & & \\
\hline
\end{tabular}

Fonte: Própria autoria, 2019. * Fatores diversos= clima agradável, polos com cobertura, mais aparelhos e mais segurança. **132 participantes optaram por não responderem ao questionário de condição socioeconômica da ABEP.

De acordo com os resultados da análise multivariada (tabela 2), verificou-se que a variável de nível de AF no lazer apresentou associação significativa ao uso das AAL no sexo feminino. As mulheres consideradas inativas no lazer, tem 2,5 vezes maior chance de usar as AAL.

Tabela 2 - Análise de regressão logística referentes ao uso das academias ao ar livre entre os fatores sociodemográficos e as condições de saúde das mulheres ( $n=428$, Rio Claro SP, Brasil)

\begin{tabular}{llll}
\hline Variável & p-valor & O.R.* & IC 95\% O.R.* \\
\hline Idade & & & \\
$\quad$ 25-39 anos & -- & 1.00 & --- \\
40-59 anos & 0.35 & 1.61 & $0,58-4,45$ \\
$\quad$ 60 anos & 0.14 & 2.10 & $0,78-5,64$ \\
\hline Escolaridade & & & \\
$\quad$ Superior & -- & 1.00 & -- \\
$\quad$ Segundo completo & 0.64 & 0.82 & $0,36-1,88$ \\
$\quad$ Primeiro completo & 0.42 & 0.66 & $0,24-1,83$ \\
$\quad$ Até quarta & 0.30 & 0.63 & $0,26-1,52$ \\
$\quad$ Nenhum & 0.17 & 1.80 & $0,78-4,16$ \\
\hline Classe Econômica & & & \\
$\quad$ A e B & 0,45 & 0,66 & $0,22-1,96$ \\
C & 1,16 & 1,54 & $0,39-2,33$ \\
$\quad$ D & -- & 1,00 & -- \\
\hline AF no lazer (IPAQ) & & & \\
$\quad$ Ativo & --- & 1,00 & -- \\
$\quad$ Inativo & 0,04 & 2,17 & $1,04-4,57$ \\
\hline Uso com orientação profissional & & & \\
$\quad$ Não & --- & 1.00 & - \\
$\quad$ Sim & 0.08 & 1.61 & $0,94-2,74$ \\
\hline Fonte: Própria autoria, 2019 & &
\end{tabular}


*OR (Odds Ratio) = Razão de risco para uso; (n=363 não usam e n=65 usam). IC 95\% OR = Intervalo de 95\% de confiança para a razão de risco.

A tabela 3 apresenta diferença significativa entre os usuários e não usuários das AAL no sexo masculino para: idade, maior frequência de não usuários <60 anos $(39,90 \%)$ e usuários $\geq 60$ anos $(68,89 \%)$, escolaridade, maior frequência de não usuários que apresentam até segundo grau completo/superior $(52,52 \%)$ e de usuários que apresentaram até a $4^{\circ}$ série $(48,89 \%)$, sobre conhecer as AAL, 25,76\% dos não usuários, não conhecem as AAL.

Tabela 3 - Associação entre o uso das AAL e os fatores sociodemográficos e as condições de saúde dos homens ( $n=243$, Rio Claro -SP, Brasil)

\begin{tabular}{|c|c|c|c|c|}
\hline Variáveis & $\begin{array}{l}\text { Não } \\
\mathbf{N}(\%)\end{array}$ & $\begin{array}{l}\text { Sim } \\
\mathbf{N}(\%)\end{array}$ & $\mathrm{x}^{2}$ & $\begin{array}{l}\text { p- } \\
\text { valor }\end{array}$ \\
\hline Faixa Etária & & & 9,38 & 0,01 \\
\hline 25-39 anos & $28(14,14)$ & $1(2,22)$ & & \\
\hline $40-59$ anos & $79(39,90)$ & $12(28,89)$ & & \\
\hline$\geq 60$ anos & $91(45,96)$ & $31(68,89)$ & & \\
\hline Escolaridade & & & 12,3 & 0,01 \\
\hline Até a $4^{\circ}$ série & $62(31,31)$ & $22(48,89)$ & & \\
\hline $2^{\circ}$ grau incompleto & $32(16,16)$ & $7(15,56)$ & & \\
\hline $2^{\circ} \quad$ grau & $104(52,52)$ & $16(35,56)$ & & \\
\hline \multicolumn{5}{|l|}{ completo/superior } \\
\hline Classe Econômica (ABEP) & & & - & 0,66 \\
\hline $\mathrm{A}$ e B & $32(24,25)$ & $9(26,47)$ & & \\
\hline $\mathrm{C}$ & $90(68,18)$ & $21(52,94)$ & & \\
\hline $\mathrm{D}$ & $10(7,58)$ & $4(11,56)$ & & \\
\hline AF no lazer (IPAQ) & & & 0,40 & 0,52 \\
\hline Ativo & $65(32,83)$ & $17(37,78)$ & & \\
\hline Inativo & $133(67,17)$ & $28(62,22)$ & & \\
\hline Possui DCNT & & & 0,05 & 0,81 \\
\hline Não & $14(17,10)$ & $31(19,30)$ & & \\
\hline Sim & $130(80,70)$ & $68(82,90)$ & & \\
\hline Conhece as AAL & & & 84,57 & 0,001 \\
\hline Não & $51(25,76)$ & $0(0,01)$ & & \\
\hline Sim & $147(74,24)$ & $45(99,99)$ & & \\
\hline Necessário para o uso das & & & - & 0,88 \\
\hline \multicolumn{5}{|l|}{ AAL } \\
\hline Companhia & $4(2,02)$ & $0(0,01)$ & & \\
\hline Tempo & $25(12,63)$ & $7(15,56)$ & & \\
\hline Não tem interesse & $67(33,84)$ & $15(33,33)$ & & \\
\hline Orientação profissional & $40(20,20)$ & $10(22,22)$ & & \\
\hline Outros Fatores & $62(31,31)$ & $11(28,89)$ & & \\
\hline
\end{tabular}

Fonte: Própria autoria, 2019. 
*Fatores diversos= clima agradável, polos com cobertura, mais aparelhos e mais segurança. **77 participantes optaram por não responderem ao questionário de condição socioeconômica da ABEP.

A tabela 4 aponta que as variáveis idade e uso com orientação profissional apresentaram associação significativa ( $p$-valor=0,05) em relação ao uso das AAL no sexo masculino. Os homens com maior chance de uso das AAL foram os que apresentaram idade $>60$ anos, sendo que a cada 1 ano de idade a chance de uso das AAL aumenta 4,7\%, e com orientação profissional a chance é $2,2 \%$ vezes maior.

Tabela 4 - Análise de regressão logística referentes ao uso das academias ao ar livre entre os fatores sociodemográficos e as condições de saúde dos homens ( $n=243$, Rio Claro -SP, Brasil)

\begin{tabular}{|c|c|c|c|}
\hline Variável & p-valor & O.R.* & IC $95 \%$ O.R.* \\
\hline \multicolumn{4}{|l|}{ Idade } \\
\hline 25-39 anos & --- & 1.00 & --- \\
\hline $40-59$ anos & 0,15 & 4.61 & $0.58-36.83$ \\
\hline$\geq 60$ anos & 0,03 & 9.54 & $1.25-73.01$ \\
\hline \multicolumn{4}{|l|}{ Escolaridade } \\
\hline Superior & --- & 1.00 & --- \\
\hline Segundo completo & 0,66 & 0.79 & $0,28-2,28$ \\
\hline Primeiro completo & 0,68 & 1.26 & $0,41-3,82$ \\
\hline Até quarta & 0,79 & 1.15 & $0,40-3,35$ \\
\hline Nenhum & 0,01 & 3.66 & $1,34-10,01$ \\
\hline \multicolumn{4}{|l|}{ Classe Econômica } \\
\hline$A$ e $B$ & 0,61 & 0,70 & $0,18-2,78$ \\
\hline $\mathrm{C}$ & 0,95 & 1,23 & $0,32-4,71$ \\
\hline $\mathrm{D}$ & --- & 1,00 & --- \\
\hline \multicolumn{4}{|l|}{ IPAQ lazer } \\
\hline Inativo & --- & 1,00 & --- \\
\hline Ativo & 0,52 & 1.24 & $0,64-2,43$ \\
\hline \multicolumn{4}{|c|}{ Uso com orientação profissional } \\
\hline Não & --- & 1,00 & --- \\
\hline Sim & 0,05 & 1,91 & $0,99-3,70$ \\
\hline
\end{tabular}

\section{Discussão}

De maneira geral, os resultados indicam que os fatores sociodemográficos e as condições de saúde de homens e mulheres (participantes deste estudo) usuários e não usuários das AAL, são semelhantes. O resultado que despertou a atenção dos autores foi a alta prevalência de doenças crônicas e a inatividade física em ambos os sexos, com baixa aderência para o uso das 
AAL. Diante do exposto, a discussão do presente estudo será mediada por possíveis alternativas que visem a efetividade e a maior utilização desses espaços. Para que que as políticas públicas de promoção da saúde sejam efetivas, se faz necessário entender esta problemática e apresentar possíveis estratégias para a melhor e/ou maior utilização desses espaços.

Neste estudo buscou-se identificar os fatores individuais e ambientais associados à pratica de $\mathrm{AF}$ em parques e praças de Curitiba-PR, evidenciando que a companhia e a prática de $\mathrm{AF}$, obtendo associação direta com a frequência (maior ou igual a uma vez por semana) aos locais públicos ${ }^{11}$.

Já neste outro estudo, revelaram que os maiores facilitadores para a prática de AF em parques públicos foram beleza, localização geográfica e infraestrutura dos parques. Por outro lado, as barreiras foram o clima e a qualidade do $\mathrm{ar}^{12}$. A dificuldade das pessoas de incorporar a prática de $\mathrm{AF}$ no seu cotidiano nas estações mais frias e chuvosas podem ser os fatores que influenciaram na menor frequência de indivíduos que utilizam as $\mathrm{AAL}^{13}$. Com relação ao clima e à qualidade do ar, são variáveis dificilmente controláveis, entretanto, são sugeridas a disponibilização de áreas cobertas e protegidas, como alternativa para evitar interrupções na regularidade da prática de $\mathrm{AF}$ em ambientes públicos ${ }^{14}$. Os autores observaram ainda que a população com menor renda pode ser motivada à pratica de $\mathrm{AF}$ em locais públicos em decorrência da presença de equipamentos gratuitos.

Conforme as análises deste estudo, evidenciou-se que a percepção do ambiente pode ser um fator de influência para a utilização de parques urbanos para a prática de AF. Além da infraestrutura, mencionada anteriormente como possível fator motivador para a prática de AF em espaços públicos, apontam também a necessidade de orientação para praticar AF, cartazes informativos e profissionais de Educação Física ${ }^{11,12,14}$.

No que se refere as AAL, as mesmas possuem cores vibrantes que, em muitos casos, podem despertar o interesse das pessoas, podendo justificar o achado do presente estudo, em que a maior parte dos respondentes conhecem as AAL, porém apenas 15,18\% e 18,51\% de mulheres e homens, respectivamente, utilizavam as AAL, sugerindo que as cores vibrantes chamam a atenção, porém não é fator determinante para a utilização das AAL. Ibiapina et al. (2017) questionam a questão das cores e afirmam que a aderência não deve somente à atratividade do local, mas também às questões específicas, como a presença ou não de um profissional de Educação Física, o qual desencadearia maior interesse por parte da população. Os mesmos autores argumentam que, embora os locais sejam públicos, em muitos casos, a 
população não frequenta estes locais, devido à falta de profissionais capacitados e, consequentemente, ao receio de não obter uma adequada orientação quanto a realização dos exercícios $^{16}$

Embora haja campanhas de conscientizações e as pessoas entendam a importância da prática de $\mathrm{AF}$, ainda faltam políticas públicas para fomentar a presença de profissionais de Educação Física nas AAL, o que pode proporcionar menor presença da população e diminuir o potencial que as AAL possuem ${ }^{17}$.

Em relação a presença de profissionais presentes nestes espaços afim de orientar os usuários, outro estudo averiguou quais os motivos levam idosos a frequentarem tais locais e sugere, a partir dos resultados obtidos, a ampliação do corpo docente presente nestes locais, assim como mencionado anteriormente ${ }^{18}$. Além disso, elencam o incentivo destes profissionais de suma importância para a população idosa, a qual se sente mais segura e motivada ${ }^{18}$. Outro ponto citado pelos autores, diz respeito a melhoria na infraestrutura destes locais e atentam para o fato de que é necessária maior atenção por parte dos governos em relação às AAL, implementando estratégias de maior adesão e investimentos mais robustos, em prol de recursos permanentes para investimentos nestes locais ${ }^{18}$.

Ainda argumentam que a motivação intrínseca da população, especialmente, a idosa, se deve à fatores pessoais e também aqueles relacionados ao local em que estão frequentando. Os autores defendem ser este um argumento plausível no incentivo da criação de novas políticas públicas, com objetivos mais centralizados nas AAL, como um aporte no fortalecimento da promoção da saúde, promovendo melhorias na qualidade de vida da população ${ }^{18}$. Demais estudos também destacam maior utilização desses espaços por idosos ${ }^{19,20,21}$.

Embora não seja o foco deste trabalho, a população mais jovem também tem apresentado altos níveis de sedentarismo (cerca de 10 horas por dia em atividades que caracterizam comportamentos sedentários $)^{22}$. Cabe destacar que o comportamento sedentário também tem sido caracterizado como fator de risco para doenças cardiometabólicas em crianças e adolescentes $^{23}$. Dados preocupantes que vêm chamando a atenção de estudiosos da área. Pessoas que gastam muito tempo em comportamentos sedentários são mais propensos à morbidade e mortalidade ${ }^{24}$. Dessa forma, é cabível pensar em medidas que aproveitem esses espaços para inserir e promover a prática de AF para a população mais jovem.

O presente estudos traz algumas limitações como, o sorteio dos setores censitários de forma conglomerada (ímpar ou par) ao invés do sorteio dos setores de forma separada, porém, 
não foi apresentada diferenças entre os setores ímpares e pares quando comparado ao Índice Paulista de Vulnerabilidade Social, e o desenho transversal, uma vez que não permite estabelecer relações de causa e efeito.

A cidade de Rio Claro-SP ainda conta com três faculdades que oferecem graduação em Educação física, uma vez que sejam aderidas as alternativas acima, é imprescindível a presença de um profissional para acompanhar, orientar e direcionar diferentes populações (faixas etárias) com necessidades e limitações distintas, assim, futuros estudos poderão ser realizados afim de verificar novamente o perfil dos usuários das AAL e a frequência de uso dos mesmos em relação ao nível de AF e saúde.

Por fim, uma política pública efetiva se dá por meio avaliações que mensurem seus efeitos com possibilidades de melhorá-las.

\section{Conclusão}

Conclui-se que conforme a Proposta da Política Nacional de Promoção da Saúde e o Plano de Enfrentamento das Doenças e Agravos Não-Transmissíveis, o uso desses espaços não está sendo efetivo e nem atendendo as necessidades de diferentes faixas etárias, pois a maior prevalência de inatividade física entre os usuários (homens e mulheres), bem como a baixa adesão ao uso desses espaços apontam que novas medidas sejam tomadas afim de tornar tal proposta mais efetiva.

\section{Referências}

1. Carta de Ottawa. Ottawa, Canadá: 1986 [acesso em 28 jan 2019]. Disponível em: http://bvsms.saude.gov.br/bvs/publicacoes/carta_ottawa.pdf.

2. Malta DC, Silva JB. Policies to promote physical activity in Brazil. Lancet; 2012;380(9838):195-6.

3. Hino AAF, Reis RS, Ribeiro IC, Parra DC, Brownson RC, Fermino RC. Using observational methods to evaluate public open spaces and physical activity in Brazil. J Phys Act Health;2010;7(2):146-54.

4. Bowler DE, Buyung-Ali LM, Knight TM, Pullin, AS. A systematic review of evidence for the added benefits to health of exposure to natural environments. BMC;2010;10:456-65. 
5. Prefeitura Municipal da Cidade de Curitiba. Secretaria Municipal de Esporte, Lazer e Juventude (SMELJ). Curitiba: 2017 [acesso em 28 jan 2019]. Disponível em: http://www.curitiba.pr.gov.br/conteudo/academia-ao-ar-livre-smelj/144.

6. Academias ao ar livre sendo reformadas. Imprensa de Rio Claro: 20 de janeiro de 2018 [acesso em 28 jan 2019]. Disponível em: http://imprensa.rioclaro.sp.gov.br/?p=43760.

7.BRASIL. Instituto Brasileiro de Geografia e Estatística. Resultados do Censo 2010: distribuição da população por sexo, segundo os grupos de idade. Rio Claro-SP: 2010. [acesso em 28 jan 2019]. Disponível em: https://brasilemsintese.ibge.gov.br/populacao/populacaopor-sexo-e-grupo-de-idade-2010.html

8.McKenzie TL, Deborah A. (2006). System for Observing Play and Recreation in Communities. [publicação online]; 2006 [acesso em 20 dez 2018] Disponível em: https://activelivingresearch.org/soparc-system-observing-play-and-recreation-communities.

9. Dumith SC. Physical activity in Brazil: a systematic review. Cad Saude Publica;2009;(3):615-26.

10. The SAS System for Windows (Statistical Analysis System), versão 9.2. SAS Institute Inc, 2002-2008, Cary, NC, USA.

11. Fermino RC, Reis RS, Individual, environmental and social variables associated with the use of public open spaces for physical activity practices: a systematic review. Rev Bras Ativ Fis e Saúde; 2013;18(5):523-35.

12. Copetti J, Neutzling MB, Silva MC. Barreiras à prática de atividades físicas em adolescentes de uma cidade do sul do Brasil. Rev Bras Ativ Fis e Saúde; 2012;15(2):88-94.

13. Santos SC, Knijnik JD. Motivos de adesão à prática de atividade física na vida adulta intermediária. Rev. Mackenzie Educ. Fís; 2006;5(1):23-34.

14. Reis RS., Cassou AC. Fatores individuais e ambientais associados ao uso de parques e praças por adultos de Curitiba-PR, Brasil. Rev Bras Cineantropom Desempenho Hum; 2012;14(4):377-89

15. Kunzler MR, Rocha ES, Bombach GD, Neves D, Santos GS, Carpes FP. Saúde no parque: características de praticantes de caminhada em espaços públicos de lazer. Saúde debat; 2014;38(102):646-53.

16. Ibiapina ARL, Moura MN, Santiago MLE, Moura NB. Characterization of users and usage pattern of outdoor fitness equipment. Rev Bras Promoç Saúde. 2017;30(4):1-10.

17. Pieri BF. Motivação para a prática de exercícios físicos em academias ao ar livre em praças públicas. Trabalho de Conclusão de Curso. Universidade Estadual de Campinas; 2015.

18. Salin MS, Virtuoso JF, Nepomuceno ASN, Weiers GG, Mazo GZ. Golden Age Gym: reasons for entry, permanence and satisfaction among participating older adults. Rev Bras Cineantropom Desempenho Hum. 2014;16(2):152-60. 
19. Hallal PC, Tenório MCM, Tassitano RM, Reis RS, Carvalho YM, Cruz DKA. Avaliação do programa de promoção da atividade física Academia da Cidade de Recife, Pernambuco, Brasil: percepções de usuários e não-usuários. Cad Saude Publica; 2010;26(1):70-8.

20. Souza CA, Fermino RC, Añes CRR, Reis RS. Perfil dos frequentadores e padrão de uso das academias ao ar livre em bairros de baixa e alta renda de Curitiba-PR. Rev Bras Ativ Fis Saúde; 2014;19(1):86-97.

21. Lepsen AM, Da Silva MC. Profile of individuals attending fitness zones in Pelotas cityRS. Rev Bras Ativ Fis Saúde; 2015; 20(4):413-24.

22. Saunders TJ, Chaput JP, Tremblay MS. Sedentary behaviour as an emerging risk factor for cardiometabolic diseases in children and youth. Can J Diabetes; 2014;38(1):53-61.

23. Ramos DE, Bueno MRO, Vignadelli LZ, Werneck AO, Ronque ERV, Coelho-E-Silva MJ. Pattern of sedentary behavior in brazilian adolescents. Rev Bras Ati Fis Saúde; 2018;23:1-9.

24. Young DR, Hivert MF, Alhassan S, Camhi SM, Ferguson JF, Katzmarzyk PT. Sedentary behavior and cardiovascular morbidity and mortality: a science advisory from the american heart association. Circulation; 2016;34(13):262-79. 